Erschienen in: Konopka, Marek/Strecker, Bruno (Hrsg.): Deutsche Grammatik Regeln, Normen, Sprachgebrauch. - Berlin, New York: de Gruyter, 2009. S.

274-306. (Institut für Deutsche Sprache. Jahrbuch 2008), https://doi.org/10.1515/9783110217360.3.274

\author{
EVA BREINDL
}

\title{
Fehler mit System und Fehler im System Topologische Varianten bei Konnektoren
}

\begin{abstract}
1
kein fehler im system kein efhler im system kein ehfler im system kein ehlfer im system kein ehlefr im system kein ehlerf im system kein ehleri fm system kein ehleri mf system kein ehleri ms fystem kein ehleri ms yfstem kein ehleri ms ysftem kein ehleri ms ystfem kein ehleri ms ystefm kein ehleri ms ystemf fkei nehler im system kfei nehler im system kefi nehler im system keif nehler im system kein fehler im system
\end{abstract}

2

kein fehler im system kein fehler imt sysem kein fehler itm sysem kein fehler tmi sysem kein fehler tim sysem kein fehler mti sysem kein fehler mit sysem

\section{3}

kein system im fehler kein system mir fehle keiner fehl im system keim in systemfehler sein kystem im fehler ein fehkler im system seine kehl im fyrsten ein symfehler im sekt kein symmet is fehler sey festh kleinr mine

Eugen Gomringer: 3 variationen zu „,kein fehler im system“

Aus: Eugen Gomringer Konkrete Poesie, Stuttgart 1978, Reclam

\begin{abstract}
Adverbkonnektoren und die von konjunktionalen Konnektoren eingeleiteten Sätze (sententiale Adverbiale) sind in der Regel äußerst stellungsflexibel. Die topologischen Varianten sind jedoch großteils nicht äquivalent, sondern mit bestimmten diskusfunktionalen und informationsstrukturellen Eigenschaften verbunden. Am Beispiel von Konnektoren in der linksperipheren Position der „Nullstelle“ (,Vorvorfeld“, ,linkes Außenfeld“) wird gezeigt, dass diese Position unabhängig von der syntaktischen Subklasse des Konnektors syntaktisch und funktional einheitlich erklärt werden kann und dass die dort auftretenden Restriktionen für Konnektoren identisch sind mit denen von V2-Komplementsatz-Einbettung unter Matrixsatzprädikate. Ein Phänomen wie ,weil mit Verbzweitstellung“ kann dadurch in einen übergeordneten Zusammenhang eingebettet werden.

Neben dieser systematischen Variation gibt es vereinzelt aber auch eine - historisch bedingte - unsystematische und nicht funktional genutzte topologische Variation, die dadurch
\end{abstract}


entsteht, dass Sprecher bei einer uneindeutigen und „schwierigen“ Ausgangslage im Sprachsystem unterschiedliche Reparaturstrategien wählen. Ein Beispiel dafür ist der korrelative Konnektor sowohl ... als auch.

Gegenstand des vorliegenden Beitrags ${ }^{1}$ sind topologische Varianten bei konjunktionalen und adverbialen Satzverknüpfern, die teilweise „normativ diskriminiert" sind, also z.B. Gegenstand öffentlicher Sprachreflexion oder als Zweifelsfall in einschlägigen Sammlungen kodifiziert sind (s. Klein in diesem Band). Dabei lassen sich zwei Variationstypen unterscheiden: Typ A, der „Fehler mit System“, erzeugt systemkonforme, wenngleich vielleicht nicht oder noch nicht von allen Sprechern und in allen Registern akzeptierte Varianten, Typ B, der „Fehler im System“, erzeugt unsystematische Varianten mit eher geringen Legalisierungschancen. Charakteristisch für diesen Typ ist ein hoher Grad von Diversifizierung der Varianten mit ebenso divergenten Beurteilungen durch muttersprachliche Sprecher. Von Gomringers 3 Variationen illustrieren die ersten beiden quasi den systematischen Variationstyp den Fehler mit System oder auch das System im Fehler -, die dritte Variante den chaotischen Variationstyp.

Als Beispiel für den systemkonformen Variationstyp dient die „Nullstelle“, „Vorvorfeld“ oder „linkes Außenfeld“ genannte satzperiphere Position, die Konnektoren unterschiedlicher syntaktischer und semantischer Klassen enthalten kann. Die leitende Fragestellung ist, inwieweit diese Position trotz variabler Besetzung satzsyntaktisch und funktional einheitlich charakterisiert werden kann. ${ }^{2}$

Den unsystematischen Variationstyp illustriert der zweiteilige Konjunktor sowohl ... als auch, der in einer großen Bandbreite von Konstruktionstypen belegt ist. Es wird gezeigt, dass hier, historisch bedingt, eine Ausgangssituation herrscht, die Sprechern eine durchgehend normgerechte Verwendung dieses Konnektors nahezu unmöglich macht.

\section{A Fehler mit System: Konnektoren an der Nullstelle}

In der Position zwischen ihren beiden Konnekten können Konnektoren unterschiedlichen syntaktischen Typs auftreten: ${ }^{3}$ a) integrierte Konjunk toren, b) des-

Für Anregungen und Kritik danke ich Stefan Engelberg, Joachim Jacobs, Anna Volodina, Gisela Zifonun sowie den Herausgebern des Bands.

2 Ein solches Bemühen kann durchaus auch als Versuch einer Antwort auf Kritik von Seiten der Gesprächsforschung verstanden werden, die die Möglichkeit einer adäquaten deskriptiven und explanativen Erfassung dieser Position mit den Kategorien einer vermeintlich präskriptiven satz- und schriftsprachzentrierten Grammatik verneint und die Alternative in einer prozessorientierten, interaktionsbasierten Kategorienbildung sieht (s. Auer 1998b, Fiehler et al. 2004, Schröder 2006).

3 Bezogen auf die syntaktische Subklassifikation des HDK (= Pasch et al. 2003) heißt das: alle Konnektorenklassen können hier auftreten. Nur hier möglich sind die Konjunktoren und Postponierer sowie einige ,syntaktische Einzelgänger“ (denn, außer, es sei denn), 
integrierte Konjunktoren, c) Adverbkonnektoren, d) VL-Subjunktoren, e) V2Subjunktoren. (Die Termini sind z.T. Arbeitstermini und dienen der Unterscheidung.) Ferner können hier Ausdrücke aus dem Nennwortschatz auftreten (Nomina wie Einwand, Problem, Grund, Fazit, finite Verbalphrasen wie es sei denn, hinzu kommt), die in dieser Position die semantische Funktion von Konnektoren haben können (S. 1 f).

(1a) In der Badstraße gibt es ein billiges Hotel aber/und [es gibt] nur wenige Restaurants.

(1b) In der Badstraße gibt es ein billiges Hotel. Aber/Und: Da will keiner wohnen.

(1c) In der Badstraße gibt es ein billiges Hotel. Allerdings, da will auch keiner wohnen.

(1d) In der Badstraße gibt es ein billiges Hotel, wobei da ohnehin keiner wohnen will.

(1e) In der Badstraße gibt es ein billiges Hotel. Wobei, wer will da schon wohnen?

(1f) In der Badstraße gibt es ein billiges Hotel. Problem: wer will da schon wohnen?

\section{Syntaktische Struktur}

Als Erstes stellt sich hier die Frage, ob der zwischen seinen Konnekten stehende Konnektor in den Beispielen (1a) bis (1f) jeweils in der gleichen syntaktischen Strukturposition steht. Unter Berücksichtigung grammatischer Intuitionen ist es sinnvoll, diese Frage in drei Teilfragen aufzuspalten, die gleichzeitig eine methodische Richtung für den weiteren Gang der Argumentation weisen.

(i) Verhalten sich integrierte Konjunktoren und desintegrierte Konjunktoren gleich?

(ii) Ist die Konjunktorstelle (1a) identisch mit den desintegrierten Positionen $(1 \mathrm{~b}, \mathrm{c}, \mathrm{e})$ ?

(iii) Lassen sich die (in einer reichhaltigen Literatur diskutierten) Unterschiede zwischen VL- und V2-Subjunktoren dahingehend erklären, dass letztere Konjunktoren sind?

In der Literatur gibt es auf diese Fragen bis heute keine kanonische Antwort, mitunter sind diesbezügliche Angaben auch uneindeutig. Von einer Position für (integrierte) Konjunktoren und desintegriertes Material gehen z. B. Bußmann (2003), Auer (1997), Pasch et al. (2003), Redder (2007) und die GDS

die traditionell den koordinierenden Konjunktionen zugerechnet werden; optional ist die Position für Subjunktoren, Verbzweitsatz-Einbetter und Adverbkonnektoren. 
(1997) aus. Bei Thim-Mabrey (1985) und in der Duden-Grammatik (2005) wird dagegen die Konjunktorstelle von einer Stelle für „Parakonjunktionen“ unterschieden. ${ }^{4}$ Uhmann (1998) analysiert mit ihrer „Koordinationshypothese" V2-weil als Konjunktor; als koordinative Verknüpfung beschreiben auch Eisenberg (1994, S. 19 ff.), Duden (2005, S. 631: „Übergang von einer unterordnenden zu einer beiordnenden Konjunktion"), Scheutz (1998) und Buscha (1989, S. 126) weil-V2-Konstruktionen, weniger eindeutig die Analyse Wegeners (1993, S. 481), sie kämen „einer parataktischen bzw. koordinierenden Satzverbindung nahe“.

Im Folgenden werden die Typen (1a) bis (1e) auf Gemeinsamkeiten und Unterschiede in einer Reihe von potentiell strukturrelevanten Eigenschaften hin überprüft.

\subsection{Prosodische Integration}

VL-Subjunktoren und integrierte Konjunktoren sind prosodisch in ihre Trägerstruktur integriert. Adverbkonnektoren, V2-Subjunktoren und desintegrierte Konjunktoren sind prosodisch mehr oder weniger abgesetzt, meist durch Pausen vor und nach dem Konnektor, durch ein nicht-progredientes Tonmuster auf dem Konnektor, vor allem aber auch durch einen Tonhöhensprung zum zweiten Konnekt (s. Auer 1998a; Fiehler et al. 2004; Volodina 2007). Unter den Subjunktoren scheint das V2-weil, anders als die etwas seltener belegten V2-Varianten von obwohl, wobei oder während noch am ehesten auf eine Pause zur Nachfolgeäußerung verzichten zu können (vermutlich in Anlehnung an das Muster von denn); doch kompensiert häufig ein Tonhöhensprung die fehlende Grenzmarkierung.

\subsection{Durchlässigkeit für epistemische Operatoren und Illokutionsoperatoren}

VL-Subjunktoren und integrierte Konjunktoren sind durchlässig für epistemische oder illokutive Operatoren aus dem ersten Konnekt, dagegen wirken alle desintegrierten Konnektoren als Stöpsel; ihr zweites Konnekt ist illokutiv selbständig. Das epistemische Satzadverb in der Beispielreihe (2) und der Frageoperator in der Beispielreihe (3) haben jeweils nur in den Verknüpfun-

4 Duden (2005, S. 900): „Parakonjunktionen“ (z. B. V2-weil); Konjunktoren „,besetzen kein eigenes Feld", sondern ,lehnen sich an das Vorfeld oder an die linke Satzklammer an"; weil, obwohl, wobei "treten auch als Konjunktionen [ohne interpunktorische Absetzung] oder Parakonjunktionen [mit Absetzung] vor Hauptsätze" (ebd. S. 1060). Auch Adverbkonnektoren im VVF sind "Parakonjunktionen“. Die GDS gibt innerhalb des „linken Außenfelds“ Stellungsabfolgen für Konnektoren, Linksversetzung, Freies Thema u.a. 
gen mit integriertem Konjunktor (2a/3a) bzw. VL-Subjunktor (2c/3b) Skopus über die gesamte Satzverknüpfung. ${ }^{5}$

(2a) Wahrscheinlich kauft sie alle Bahnhöfe und setzt ein Hotel auf die Schlossallee.

(2b) ?Wahrscheinlich kauft sie alle Bahnhöfe. Und: setzt ein Haus auf die Schlossallee.

(2c) Wahrscheinlich kauft sie die Schlossallee, weil die so lukrativ ist.

(2d) $\neq$ Wahrscheinlich kauft sie die Schlossallee. Nämlich, die ist so lukrativ.

(2e) $\neq$ Wahrscheinlich kauft sie die Schlossallee. Weil, die ist so lukrativ.

(3a) Kauft sie alle Häuser und setzt ein Hotel auf die Schlossallee?

(3b) Kauft sie die Schlossallee, weil die so lukrativ ist?

(3c) $\neq$ Kauft sie die Schlossallee? Nämlich, die ist so lukrativ.

(3d) $\neq$ Kauft sie die Schlossallee? Weil, die ist so lukrativ.

Aus ebendieser Undurchlässigkeit erklärt sich auch, weshalb desintegriertes Material zusammen mit der nachfolgenden Satzstruktur nicht unter ein Matrixsatzprädikat eingebettet werden kann. Ein V2-weil verhält sich hier genauso wie denn (s. HDK 2003, S. 590 f.).

(4a) Hans hat entdeckt, dass Anna gewonnen hat, weil sie geschummelt hat.

(4b) $\neq$ Hans hat entdeckt, dass Anna gewonnen hat, weil/denn sie hat geschummelt.

\subsection{Finitheitsmerkmale bei Subjunktoren}

Fast alle Dialekte kennen weil und obwohl mit Verbzweitstellung - es ist bekanntlich das im Substandard immer bewahrte ältere Muster (s. Sandig 1973, Selting 1999). Einige Dialekte können diese und andere Subjunktoren in der 2. Person flektieren. ${ }^{6}$ Kein Dialekt kann aber V2-Subjunktoren flektieren. Die

5 Die Akzeptabilitätsbewertungen bei den folgenden Beispielblöcken beruhen auf Introspektion, manchmal abgestützt durch das Urteil weiterer muttersprachlicher Sprecher. Eine kontrollierte Informantenbefragung zur Erhebung skalierter Bewertungen konnte aus Zeitgründen nicht durchgeführt werden, wäre aber für eine feinkörnigere Auswertung der Daten, insbesondere im B-Teil dieses Beitrags, wünschenswert. Da es für die Argumentation im Folgenden meist ausreicht, wenn ein Akzeptabilitätsgefälle von einer Variante zur anderen nachvollziehbar ist, es also immer nur um vergleichende Bewertungen im Sinne eines besser - schlechter geht, wirkt sich diese ansonsten vielleicht nicht mehr ganz zeitgemäße Methode hier nicht notwendig nachteilig aus.

6 Strittig ist, ob es sich um Klitisierung oder um Flexion bzw. „Complementizer Agreement" handelt. Überzeugende Argumente gegen Klitisierung führt Oppenrieder (1991, S. 169 ff.) ins Feld: das Subjektspronomen kann zusätzlich auftreten (so auch in den obigen Beispielen) und die Endung ist nicht immer phonologisch auf ein Subjektspronomen zurückführbar (so auch im Schwäbischen in (5)). Für die hier geführte Ar- 
V2-Varianten zum flektierten VL-obwohl des Augsburger Stadtdialekts (5a) bzw. zum flektierten weil im Mittelbairischen $(5 \mathrm{c})$ sind nach übereinstimmender Meinung von Dialektsprechern nicht akzeptabel.

(5a) Meldsch du di zur Prüfung an, obwohlsch du des Buch gar net glesa hosch?

(5b) *Meld di doch zur Prüfung an, obwohlsch, du hosch ja das Buch gar net glesa!

(5c) Mià sàn àlloàns hoàmgangà, weis ees need kemà seids. (Merkle 1993, S. 189)

(5d) *Mià sàn àlloàns hoàmgangà, weis ees seids need kemà.

Die Konjunktionsflexion gilt gemeinhin als Argument für identische Strukturposition von Verbletztsatz-Einleiter und Finitum im Verbzweitsatz, die C(OMP)-Position. V2-Subjunktoren stehen offensichtlich nicht in dieser Strukturposition.

\subsection{Vorwärtsellipsen (Analepsen) bei Konjunktoren und Subjunktoren}

VL-Subjunktoren und integrierte Konjunktoren lassen sogenannte „Vorwärtsellipsen" im 2. Konnekt zu, d. h. Ellipsen von Material, das sie mit dem ersten Konnekt teilen. Bei Subjunktoren handelt es sich dabei um sogenannte „Subjekt-Kopula-Ellipsen“ (s. HdK 2003, S. 364 f.), bei Konjunktoren um Koordinationsreduktionen und Gapping. V2-Subjunktoren, desintegrierte Konjunktoren und Adverbkonnektoren blockieren Vorwärtsellipsen und erzeugen Strukturen von geringerer Akzeptabilität.

(6a) Hans ist nicht oft erkältet, obwohl meist ohne Jacke draußen.

(6b) *Hans erkältet sich nicht leicht, obwohl, meist ohne Jacke draußen.

(7a) Hans hat Anna Blumen mitgebracht und ihrem Mann eine Flasche Rotwein.

(7b) ?Hans hat Anna Blumen mitgebracht. Und: ihrem Mann eine Flasche Rotwein.

(7c) ?Hans hat Anna Blumen mitgebracht. Außerdem: ihrem Mann eine Flasche Rotwein.

gumentation kann die Frage vielleicht auch offen bleiben: Wie Wegener (1993, S. 295) gezeigt hat, kann auch Enklise von Pronomina nur an VL-Subjunktoren, nicht aber an V2-Subjunktoren erfolgen: vgl. [...] weil's ihm empfohlen wurde vs. ?weil's wurde ihm empfohlen. 


\subsection{Interpretationseffekte bei Konjunktoren}

Der Konjunktor und kann kontextgesteuert interpretativ ,angereichert " werden (s. Breind1 2007): konditional wie in (8a), ,implikativ“ wie in (9a) (s. Reis 1993), sequentiell bzw. konsequentiell wie in (10a) u. a. m. Desintegration verhindert solche Anreicherungen und erlaubt nur die adverbtypische Listeninterpretation, außerdem gilt".

(8a) Sag das noch einmal und du kannst was erleben! (konditional)

(8b) $\neq$ Sag das noch einmal. Und: Du kannst was erleben.

(9a) Sei so lieb und reich mir den Zucker. (implikativ)

(9b) $\neq$ Sei so lieb. Und: reich mir den Zucker.

(10a) Es regnete tagelang und die Flüsse traten über die Ufer. [,und dann/und infolgedessen']

(10b) $\neq$ Es regnete tagelang. Und: die Flüsse traten über die Ufer.

\subsection{Koordinierbarkeit von Material in der Nullstelle}

Satzstrukturen mit desintegriertem Material sind im Unterschied zu ihren integrierten Pendants nicht in der Weise koordinierbar, dass das desintegrierte Material Bestandteil der Koordinate ist. Dieselbe Beschränkung gilt wieder für das im HDK als syntaktischer Einzelgänger klassifizierte begründende denn.

(11a) Hans ist heute zu Hause, weil er noch arbeiten will und weil seine Frau krank ist.

(11a) *Hans muss zu Hause sein, weil, das Licht ist an und weil, das Auto steht vor der Tür.

(11c) *Hans muss zu Hause sein, denn das Licht ist an und denn das Auto steht vor der Tür.

(12a) *Ich muss heute noch Klausuren korrigieren. Deswegen, ich bleibe zu Hause und deswegen: Fritz kriegt meine Konzertkarte.

(12b) Hans muss heute Klausuren korrigieren. Deswegen ist er heute zu Hause und deswegen hat er auch seine Konzertkarte an Fritz verkauft.

Die nachstehende Tabelle fasst die Ergebnisse zusammen. Die Werteverteilung zeigt eine klare Trennung zwischen integrierten und desintegrierten Konnektoren, für die die ursprüngliche Wortartzugehörigkeit des Konnektors keine Rolle spielt. 


\begin{tabular}{|c|c|c|c|c|c|}
\hline & \multicolumn{2}{|c|}{ integriert } & \multicolumn{3}{|c|}{ desintegriert } \\
\hline & $\begin{array}{l}\text { integrierter } \\
\text { Konjunktor }\end{array}$ & $\begin{array}{l}\text { VL-Sub- } \\
\text { junktor }\end{array}$ & $\begin{array}{l}\text { V2-Sub- } \\
\text { junktor }\end{array}$ & $\begin{array}{l}\text { desinte- } \\
\text { grierter } \\
\text { Konjunktor }\end{array}$ & $\begin{array}{l}\text { Adverb- } \\
\text { konnektor }\end{array}$ \\
\hline $\begin{array}{l}\text { 1. prosodische } \\
\text { Integration }\end{array}$ & + & + & - & - & - \\
\hline 2. Durchlässigkeit & + & + & - & - & - \\
\hline $\begin{array}{l}\text { 3. Konjunktional- } \\
\text { flexion }\end{array}$ & & + & - & & \\
\hline 4. Vorwärts-Ellipsen & + & + & - & eher - & eher - \\
\hline $\begin{array}{l}\text { 5. Uminterpreta- } \\
\text { tionen }\end{array}$ & + & & & - & \\
\hline 6. Koordinierbarkeit & & + & - & & - \\
\hline
\end{tabular}

Damit kann für die syntaktische Struktur ein erstes Zwischenfazit gezogen werden.

(i) Desintegrierte Subjunktoren und Konjunktoren verhalten sich an der Nullstelle anders als ihre integrierten Pendants und eher so wie die nur parataktisch verknüpfenden Adverbkonnektoren. Ihre angestammten Wortarteigenschaften werden also zumindest teilweise überschrieben.

(ii) Der auf einen Konnektor in der Nullstelle folgende Satz muss syntaktisch und illokutionär selbständig sein und es darf nicht von außen in ihn ,hineinregiert" werden; m.a.W., es gilt das ,island constraint".

(iii) Es ist sinnvoll, zwei Strukturstellen für Konnektoren vor dem Vorfeld eines Verbzweitsatzes zu unterscheiden: eine „Koordinatorstelle“ und eine „Nullstelle“. Erstere ist eine nur für Konjunktoren reservierte Position, sie ist an koordinative Verknüpfung gekoppelt (also auch an die Zulässigkeit von Koordinationsreduktionen) und Bestandteil der Satzstruktur. Ihre syntaktische Modellierung ist stark theorieabhängig (z. B. als Kopf einer Koordinatorphrase bei Wöllstein 2008 und Johannessen 1998; dagegen bei Eisenberg (1999, S. 372) strukturell keinem der Koordinate zugeordnet). In die desintegrierte Nullstelle kann Material verschiedenster Klassen treten, einschließlich der Konjunktoren selbst. Sie ist nicht Teil des nachfolgenden Satzes. Die Verknüpfung ist nicht koordinierend, sondern nur parataktisch und Kohärenz wird nur semantisch ohne oberflächliche Kohäsionsverfahren hergestellt.

Diese Analyse weicht insofern von der Koordinationsanalyse für V2-Subjunktoren (Uhmann 1998) ab, als sie erstens V2-Subjunktoren mit desintegrierten Adverbkonnektoren und desintegrierten Konjunktoren selbst parallelisiert und damit in einen größeren Zusammenhang stellt -, und zweitens allenfalls mit einer Teilklasse der traditionell zu den koordinierenden Konjunktionen ge- 
rechneten Einheiten Analogien sieht, nämlich mit „Satzkonjunktoren“ wie denn, außer, es sei denn (die nach der restriktiveren Auffassung des HDK von Koordination eben nicht zu den koordinierenden Konjunktionen gehören), nicht aber mit den echt koordinierenden Konjunktionen und und oder (auf die von und und oder abweichende Beschränkung in Format und Anzahl der Koordinate bei V2-Subjunktoren weist Uhmann selbst hin). Die Blockierungsphänomene, der „Inselstatus“ von Sätzen nach desintegriertem Material und ihre illokutive Selbständigkeit legen m.E. aber eine Unterscheidung von Koordination und Parataxe nahe. V2-Subjunktoren und desintegrierte Konjunktoren nehmen die Eigenschaften der parataktisch verknüpfenden Adverbkonnektoren an, die ja nach mehrheitlicher Meinung auch nicht zu den koordinierenden Konjunktionen zu rechnen sind, (wenngleich die traditionelle Bezeichnung „Konjunktionaladverb“ natürlich auf ihre Zwittrigkeit abhebt).

Was bei dieser Analyse noch zu erklären bleibt, ist zum einen die komplementäre Distribution von Konjunktoren und V2-Subjunktoren, die, wie Uhmann (1998) bemerkt, gut zur Annahme identischer Strukturposition von V2-Subjunktoren und Konjunktoren passt. Zum anderen wirkt es befremdlich, dass die (integrierte) Koordinatorposition vor der (desintegrierten) Nullposition erscheinen soll. Dieser Fall tritt scheinbar auf bei der Kombination von Konjunktoren und desintegrierten Adverbkonnektoren in der Nullposition, wo sie eben nicht strikt komplementär verteilt sind. Solche Kombination unterliegen allerdings semantischen Restriktionen und die Konstruktion hat insgesamt dann die oben beschriebenen Eigenschaften der desintegrierten Strukturen, d.h., alle bei integrierten Konjunktoren möglichen koordinationstypischen Effekte sind blockiert. Es ist also davon auszugehen, dass Konjunktor und desintegrierter Adverbkonnektor eine komplexe Einheit in der Nullstelle bilden.

(13) Die Schlossallee ist ein teures Pflaster. Und außerdem/und obendrein/und schließlich/und im Übrigen/und daher/und doch/aber andererseits/aber dennoch/aber trotzdem/oder anders gesagt: Da kann ich mir kein Hotel leisten.

(14) *aber ferner, *und hingegen, *und allerdings, *und nämlich, *aber nämlich, *oder nämlich, *oder allerdings, ?aber daher: Da kann ich mir kein Hotel leisten.

Die semantischen Regularitäten hinter dieser Kombinatorik sind noch weitgehend unklar; Kombinationen innerhalb derselben oder benachbarter semantischer Klasse (additiv: und außerdem; metakommunikativ: oder mit anderen Worten; adversativ-konzessiv: aber dennoch) sind meist möglich solche „Doppelmoppelungen" treten auch satzintegriert auf (weil nämlich, wohingegen aber, sodass also), allerdings gibt es bei den adversativen Einschränkungen (*aber allerdings, *aber hingegen, *aber jedoch). Und scheint insgesamt kombinationstoleranter als aber und oder und kann auch zusammen mit konzessiven (und dennoch, und trotzdem, und doch) auftreten, jedoch 
nicht mit adversativen (*und hingegen, *und allerdings, *und jedoch). Generell haben solche Kombinationen an der Nullstelle eine Tendenz zur phraseologischen Verfestigung. Das konzessive und doch beispielsweise ist weder mit einem und noch mit einem doch an dieser Stelle äquivalent (vgl. auch: und zwar).

(15) Der Kampf der Maschinen ist so gewaltig, daß der Mensch fast ganz davor verschwindet. Und doch: Hinter allem steckt der Mensch. (FSP/ BDJ, S. 144)

Ausgeschlossen sind alle Kombination mit dem kausalen (Antezedens markierenden) nämlich; der zweite kausale Adverbkonnektor des Deutschen, schließlich, ist zwar an der Nullstelle mit und kombinierbar, wird dann aber nicht kausal, sondern im Sinne eines Abschlusses einer Sequenz interpretiert (zu weiteren Disambiguierungsfaktoren für schließlich s. Breindl/Waßner 2006, S. 53 f.). Damit könnte die Nicht-Kombinierbarkeit von und und V2-weil also auch semantische Gründe haben: Das eher ikonisch-sequentiell interpretierte und verträgt sich schlecht mit der antiikonischen Anordnung der nachgelieferten Begründung. Die übrigen V2-Subjunktoren des Deutschen sind adversativ (während, wogegen) bzw. genuin konzessiv mit der adversativen ,allerdings'-Interpretation als V2-Subjunktoren (wobei, obwoht). Verstärkende Kombinationen von aber mit adversativen Adverbkonnektoren in Juxtaposition an der Nullstelle sind aber, wie eben dargelegt, ebenso eingeschränkt wie Kombinationen der adversativen Adverbkonnektoren mit und.

Der Ausschluss einer Kombination von Konjunktor und V2-Subjunktor an der Nullstelle darf nicht verwechselt werden mit der in 1.6 erwähnten generellen Nicht-Koordinierbarkeit von desintegriertem Material. Auch aus dieser Restriktion folgt m.E. nicht zwangsläufig, dass sich die koordinierende Konjunktion und das desintegrierte Material in derselben Strukturposition befinden; vielmehr könnte sie auch auf eine generelle Beschränkung von syntaktischen Verkettungsverfahren beim Aufbau komplexer Satzstrukturen zurückgeführt werden: Eine parataktisch verknüpfte komplexe Struktur [p q] kann ihrerseits weder unter eine andere Struktur eingebettet, noch kann sie mit einer anderen parataktisch verknüpften Struktur koordiniert werden.

\section{Semantische Restriktionen für Material an der Nullstelle}

Die in der Literatur speziell zu den V2-Subjunktoren übliche methodische Herangehensweise ist die Sichtung und Sortierung einer Fülle von Belegen. Hier wird ein anderer Weg gewählt: aufbauend auf die bekannten Daten und Ergebnisse (auf die nicht im Einzelnen eingegangen werden kann) wird nach Restriktionen gesucht, in der Hoffnung, über den Negativbefund Aufschluss über die semantische Natur der Nullstelle zu erhalten. ${ }^{7}$

\footnotetext{
Die rein ,positivistische“ Herangehensweise birgt ohne das Korrektiv des Negativbefunds die Gefahr einer gewissen Beliebigkeit in der semantischen und kommunikativfunktionalen Kategorisierung der Daten und damit auch geringer Trennschärfe gegen-
} 


\subsection{Restriktionen für V2-Subjunktoren}

Das Vorkommen von V2-Subjunktoren an der Nullstelle unterliegt starken semantischen Restriktionen (s. Altmann 1997): Es ist beschränkt auf kausales weil, adversative (während(dessen), wo(hin)gegen) und konzessive Konnektoren (obwohl, obschon, wobei), welch letztere als V2-Subjunktoren wie allerdings im Sinne einer Rücknahme zu interpretieren sind.

(16) Zumindestens einer der Täter zeigte bereits am Tag nach dem Raubversuch Reue. Er begleitete das von seinem Freund mit Drohungen eingeschüchterte Opfer in Linz zur Polizei und wollte Selbstanzeige machen. „Weil, dann bekommt man eine geringere Strafe“, gestand er gestern sein Motiv. (Neue Kronen-Zeitung, 08.08.1995, S. 13)

(17) Das Sommerkorn ist sehr schlecht ausgefallen, währenddessen das mit dem Winterkorn, das ging noch einigermaßen. (Zwirner Korpus ZW1Q8)

(18) Aber des ist doch im wesentlichen des, woraus die Stadt Worms ihren Etat finanziert. Während die große Masse der sonst aufzuwendenden Steuern gehen ja entweder nach Bonn, an die Bundesregierung oder an die Länder, nach Mainz. (Pfeffer Korpus 100)

(19) Ehrlich gesagt als Winzer kann ich das behaupten, es gibt nur Trauben, die wo edelfaul werden und ein edelfauler Trauben, der hat die Brühe, wogegen ein wurmfauler Trauben, der wird schimmelig. (Zwirner Korpus ZW1P1)

(20) Österreichs Bundeskanzler Vranitzky ist auch Politiker, obschon: Körperhaltung und Diktion lassen ja mitunter vermuten, daß er das ungern ist. (Die Presse, 12.01.1996, S. 1)

Auch die nullstellenfähigen Einheiten gleichwohl, alldieweil, trotzdem, indessen, zumal fügen sich ins Bild dieser semantischen Restriktion. Sie haben aber neben einer Subjunktorverwendung auch eine Adverbverwendung, sodass hier prima facie nicht entscheidbar ist, ob es sich um V2-Subjunktor oder desintegrierten Adverbkonnektor handelt (s. Waßner i.Dr.).

(21) Es ist müßig, gegenwärtig darüber zu rätseln, ob Engholm auch der Kandidat für 1994 sein wird. Gleichwohl: die Lafontaine- Frage ist nicht ausgestanden. (die tageszeitung, 12.12.1990, S. 10)

über anderen Formkategorien. So zählt beispielsweise Schröder (2006, S. 217 f.) im Anschluss an Fiehler et al. (2004) sieben „kommunikative Leistungen“" der Vorvorfeldbesetzung auf, bei denen es sich ,nur um eine Auswahl möglicher Funktionen von Vorvorfeldbesetzungen für die Folgeeinheit" handle. Allerdings ist hier der Gegenstand weiter gefasst und umfasst beispielsweise auch Freies Thema und Linksversetzung. 
(22) Und im Oktober soll die „Goldene Stimmgabel“ wieder von hier ausgestrahlt werden, indes: Ausgerechnet hier harzt es ein bissel. (Mannheimer Morgen, 23.01.1998)

(23) Aufklärung alleine genüge nicht, zumal: „Das Thema ist hinreichend bekannt." (die tageszeitung, 08.05.1995, S. 29)

Ausgeschlossen sind kausales da (24a) und alle anderen Klassen: temporale (24b), konditionale (24c), konsekutive (24d), finale (24d) und instrumentale (24e).

(24a) *Erna pfiff vergnügt, da sie putzte die Wohnung.

(24b) *Erna pfiff vergnügt, während/sobald/als/nachdem/bevor sie putzte die Wohnung.

(24c) *Erna pfeift, wenn/falls/sofern sie putzt die Wohnung.

(24d) *Erna putzt emsig, sodass/auf dass/damit die Wohnung wird sauber.

(24e) *Die Wohnung ist blitzsauber, dadurch dass/indem Erna hat stundenlang geputzt.

\subsection{Restriktionen für Adverbkonnektoren}

Bei Adverbkonnektoren sind die Restriktionen insgesamt „weicher“, in der Tendenz aber ähnlich wie bei den V2-Subjunktoren. Weitgehend uneingeschränkt an der Nullstelle möglich sind die beiden einzigen kausalen (d.h. ihr Antezedens markierenden) Adverbkonnektoren, nämlich und schließlich, diese verknüpfen ausschließlich nicht-propositional.

(25) „Wir haben ihnen nichts versprochen, wir haben aber hinterher etwas gehalten, was wir nicht versprochen haben, nämlich der hat eine ganz schöne Hilfe für sein Land bekommen." (Spiegel 17.9.07, S. 67)

(26) Sparen wäre bei der ohnehin abgespeckten „Breminale“ kaum mehr möglich, meint Fleckenstein. Schließlich: „Welches Zelt hätten wir denn weglassen sollen?" (die tageszeitung, 16.05.1997, S. 24)

Möglich sind ferner, ganz analog zu den Beschränkungen für V2-Subjunktoren, die adversativen (allerdings, andererseits, dagegen, freilich, hingegen, jedoch, wiederum) und adversativ-restriktiven (allein, bloß, nur) sowie die konzessiven (dennoch, nichtsdestotrotz, trotzdem, dabei). Hinzu kommen bei den Adverbkonnektoren die additiven (außerdem, ferner, obendrein, überdies) und metakommunikativen (d.h., m.a.W., im Übrigen, kurzum, anders gesagt), die beide als Subjunktoren nicht ausgeprägt sind.

(27) Wenn wir, meine Frau und ich, heutzutage Arbeiten von Historikern über die Nazizeit lesen, so sagen wir uns bisweilen: „Mein Gott, der Mann hat ja keine Ahnung - allerdings, woher soll er sie auch haben? Er war ja nicht dabei. (Die Zeit, 19.06.87, S. 39) 
(28) Die Botschaft hör ich wohl, allein, mir fehlt der Glaube. (Goethe, Faust)

(29) Ich finde das albern, aber der Amerikaner freut sich'n Keks, dass er die Begrüßung auf Deutsch hinkriegt. Dabei: Viel war da nicht zu begrüßen. (die tageszeitung, 17.4.2000, S. 23)

(30) „Wir gehen gleich nebenan ins Kino, da ist's dunkel, und niemand schaut dich an, Burgl. Außerdem ... da brauchst du dich doch nicht zu schämen!“ (MK1/TJM, S. 11)

(31) Edle Pferde, schöne Frauen und reiche Männer wünscht man sich ins Stadion. Kurzum: Die Prominenz soll es sein, die diesem Gelände erst das richtige Flair verleiht. (die tageszeitung, 07.06.1991, S. 28)

Ausgeschlossen oder deutlich schlechter sind wieder temporale (abermals, dann, danach, darauf, davor, inzwischen) und die ohnehin kaum adverbial kodierenden konditionalen (andernfalls, sonst). Konsekutive (d.h. ihr Konsequens markierende) sind nur mit nicht-propositionalem Bezug möglich wie in (32a), nicht aber propositional wie in (32b). Die sowohl propositional wie nicht-propositional verknüpfenden Pronominaladverbien deshalb, deswegen, daher sind an der Nullstelle im Korpus typischerweise mit zwei Umgebungen belegt: mit nachfolgender direkter Rede oder mit einer Folgeäußerung von direktivem Illokutionstyp. Ansonsten sind hier wieder die schlussfolgernden (also ausschließlich nicht-propositionsbezogenen) Adverbkonnektoren ergo, folglich, mithin möglich.

(32a) Über den Winter wird der Reifen schlapp. Deswegen: Luftdruck checken!

(32b) ?Über den Winter wurde der Reifen schlapp. Deswegen: ich checkte den Luftdruck.

\subsection{Restriktionen für Adverbialsätze}

Desintegrierte kausale, konditionale oder finale Adverbialsätze werden überwiegend nicht-propositionsbezogen interpretiert. Desintegration ist bei diesen sogenannten Sprechaktadverbialen fast obligatorisch.

(33) Weil du danach fragst,

$\left.\begin{array}{l}\text { Wenn du mich fragst, } \\ \text { Damit du beruhigt bist, }\end{array}\right\} \begin{aligned} & \text { ich finde den Film langweilig. } \\ & \neq \text { finde ich den Film langweilig. }\end{aligned}$

Eine Ausnahme zur Vorfeldrestriktion bilden diejenigen Sprechaktadverbiale, die aufgrund ihres propositionalen Gehalts eindeutig als nicht-propositionsbezogen gekennzeichnet sind (vgl. Pittner 1999 für sententiale, Meinunger 2004 für nicht sententiale Sprechaktadverbiale). Ambige Adverbiale erhalten dagegen nur an der Nullstelle die nicht-propositionsbezogene Interpretation, im Vorfeld werden sie propositionsbezogen interpretiert. 
(34a) Nebenbei gesagt/ernsthaft gesagt, die Aufgabe macht Probleme.

$(34 \mathrm{~b})=$ Nebenbei gesagt/ernsthaft gesagt macht die Aufgabe Probleme.

(34c) = Nebenbei/ernsthaft, die Aufgabe macht Probleme.

(34d) $\neq$ Nebenbei/ernsthaft macht die Aufgabe Probleme.

Faktische Konditionalsätze sind eher desintegrierbar als Standardkonditionalsätze.

(35) Wenn Sie sagen, daß man dazu nicht in der Lage ist, wer soll Ihnen denn dann in den neuen Bundesländern noch glauben [...]. http://www.bundestag.de/bau_kunst/debatte/bdr_006.html

(36) Auch wenn das nun alles etwas negativ klingt: Journalistin zu sein ist für mich nach wie vor einer der spannendsten Berufe, den ich kenne. (http://www.ucip.ch/th/kg)

Auch für Irrelevanzkonditionalsätze ist die Desintegrationstendenz bekannt (König/van der Auwera 1988, Pittner 1999, d'Avis 2004).

(37) Wenn du auch dagegen bist,

Wie sehr du auch zeterst,

Ob du willst oder nicht,

auf dieses Konzert verzichte ich nicht!

?verzichte ich nicht auf dieses Konzert.

\subsection{Restriktionstendenzen}

In der Zusammenschau der drei Nullstellenbesetzungen kristallisieren sich Tendenzen heraus.

(i) Nicht-propositionaler Bezug erleichtert Desintegration; bei diesbezüglich ambigen Konnektoren bzw. Adverbialsätzen wird an der Nullstelle die propositionale Interpretation blockiert.

(ii) Ein Konnektor, der offenen Faktizitätswert für sein internes Argument induziert (Standardkonditionale, finale), kann nicht desintegriert auftreten. Desintegration ist auf Konnektoren beschränkt, deren internes Argument „faktisch fundiert" ist (kausale, additive, adversative, konzessive).

(iii) Ein Konnektor, der die Geltung seines internen Arguments voraussetzt (kausales $d a$ ), kann nicht desintegriert auftreten. ${ }^{8}$

8 Die manifesten Unterschiede zwischen da und weil ( $d a$-Sätze antworten nicht auf warum-Fragen, stehen nicht im Skopus höherer Operatoren, sind nicht fokussierbar, sind eher anteponiert, während weil-Sätze eher postponiert sind) sind ein ausgiebiger Diskussionsgegenstand. Sie werden zum einen auf den Unterschied propositional/ Sachverhaltsbezug vs. nicht-propositional/Äußerungsbezug zurückgeführt (HdK 2003, S. 397; Duden 2005, S. 1098), zum anderen auf einen in der deiktischen Herkunft von 
(iv) Sententiale Adverbiale können nur dann desintegriert werden, wenn sie den Wahrheitswert der nachfolgenden Struktur nicht beeinflussen.

Diese Restriktionen sind nicht zufällig und kein rein lokales Phänomen. Sie finden sich auch bei nicht-konnektoraler Besetzung der Nullstelle, nämlich bei (semantisch einstelligen) modalen Satzadverbien, - und diese sind wiederum ein Spiegel der Restriktionen bei Matrixsatzprädikaten von V2-Komplementsätzen. Auch hier gelten nämlich im Wesentlichen zwei Restriktionen (s. Reis 1997, Auer 1998b):

- keine präsupponierenden Prädikate

- keine negierenden oder modal abschwächenden Prädikate, keine Prädikate mit „nicht-faktisch fundierten“ Komplementsätzen (GDS 1997, S. 1454 ff.)

Das typische Vorkommen von V2-Komplementsätzen ist das als referierte Rede unter einem verbum dicendi. Die Analogie dazu bei den Konnektoren ist der häufige Sprechaktbezug von Konnektoren und sententialen Adverbialen an der Nullstelle, der oft in Form eines Hypersatzes mit einer propositionalisierten Illokutionskomponente dargestellt wurde (vgl. Thim-Mabrey (1985, S. 231), die Konnektoren an der Nullstelle generell als „Bestandteile eines elliptischen metakommunikativen Hypersatzes“ analysiert; s. auch GDS 1997, S. 2306).

(38) Weil du danach fragst (sage ich): ich finde den Film langweilig.

(39) Über den Winter wird der Reifen schlapp. Deswegen (rate ich): Luftdruck checken!

Ferner können V2-Komplementsätze als Alternanten zu dass-Sätzen unter Gewissheitsprädikate eingebettet werden und nach assertionsbestätigenden Satzadverbien auftreten. Ihr internes Komplement kann also, wie das der V2-Subjunktoren, als „faktisch fundiert" gelten.

(40a) Es steht fest/Tatsache ist/richtig ist, die Schlossallee ist die beste Adresse in der Stadt.

(40b) In der Tat/zweifellos/keine Frage: die Schlossallee ist die beste Adresse in der Stadt.

Faktive Verben, die die Geltung der Proposition ihres Komplementsatzes präsupponieren, sind als Matrixsatzprädikate ausgeschlossen. Ganz analog kann an der Nullstelle vor einem V2-Satz auch kein bewertendes Satzadverb auftreten. Die Analogie zu den Restriktionen bei V2-Subjunktoren besteht zum einen im Ausschluss eines kausalen V2-da, zum anderen in der informations-

da verankerten Faktor „bekannter Grund/besonders evident“ (Duden 2005, S. 638), „diskurspräsupponiert" (vs. behauptet) (Pasch 1983). Mit da präsentiert ein Sprecher eine Begründung so, als wäre sie dem Hörer bereits bekannt. 
strukturellen Beschränktheit von V2-weil und denn: beide können nicht mit einem internen Konnekt verwendet werden, das im informationsstrukturellen Hintergrund liegt oder „aufgrund von Evidenz präsuppositional ist“ (HDK 2003, S. 586).

(41a) ??Es ist bedauerlich/es missfällt mir/es freut mich: Hans hat schon wieder verloren.

(41b) ?Bedauerlicherweise/leider/zum Glück/erstaunlicherweise: Hans hat verloren.

(41c) Hans hat verloren. Wir haben schon befürchtet, dass er zu WEInen\anfängt, weil er verloren hat.

(41d) *[...] Wir haben schon befürchtet, dass er zu WEInen\ anfängt, weil er hat verloren.

(41e) * [...] Wir haben schon befürchtet, dass er zu WEInen\ anfängt, denn er hat verloren.

Als Matrixsatzprädikate von V2-Komplementsätzen sind auch negierende und modal abschwächende Prädikate ausgeschlossen, Satzadverbien der epistemischen oder deontischen Modalität können an der Nullstelle ebenfalls nicht auftreten. Das Analogon dieser Restriktion bei den Konnektoren ist der Ausschluss konditionaler und finaler V2-Subjunktoren. Diese assertieren ihr internes Komplement nicht.

(42a) *Es ist nicht der Fall/Falsch ist/Zu bestreiten ist: alle Bahnhöfe sind in Privatbesitz.

(42b) *Es ist möglich/Es kann sein/Es liegt nahe: alle vier Bahnhöfe sind in Privatbesitz.

(42c) *Keineswegs/wahrscheinlich/vielleicht: alle Bahnhöfe der Stadt sind in Privatbesitz.

(43a) *Es gehört sich/Pflicht ist/ich will: der Besitzer der Schlossallee gewinnt.

(43b) ??Unausweichlich/notwendig/zwangsläufig: wer die Schlossallee besitzt, gewinnt.

Gegen diese Beschränkung auf assertierte Komplemente gibt es allerdings sowohl von Seiten der Matrixsatzprädikate als auch von Seiten der Konnektoren ein auf den ersten Blick schwerwiegendes Gegenargument: Als Matrixsatzprädikate von V2-Sätzen können nämlich nicht nur verba dicendi, sondern auch doxastische Einstellungsprädikate (verba putandi) wie glauben, annehmen, vermuten auftreten. Umgekehrt liegen mit der kleinen Konnektorenklasse der Verbzweitsatz-Einbetter (angenommen, unterstellt, gesetzt (den Fall), vorausgesetzt) semantisch konditionale Konnektoren vor, für die 
eine Verbzweitstellung ihres internen Konnekts grammatikalisiert ist. Mit einer leichten Modifizierung lässt sich aber auch für V2-Sätze nach diesen Prädikaten bzw. Konnektoren die Assertiertheitsbedingung retten. Ein Hinweis findet sich bei Reis (1997). Sie klammert in ihrer Untersuchung von V2Komplementsätzen die unter Gewissheitsprädikaten eingebetteten aus und formuliert für die verbleibenden V2-einbettenden Prädikate den gemeinsamen Nenner so: „V2-Prädikate fixieren eine zur aktualen Welt alternative (Glaubens-, Sagens-, Präferenz-)Welt des zugehörigen Subjekts (soweit vorhanden), in der die abhängige Proposition als wahr beansprucht wird" (ebd. S. 122), V2-Komplemente hätten den Status von „vermittelten Assertionen“. Bei der Einbettung eines V2-Satzes unter ein verbum putandi wird also gerade nicht Geltungseinschränkung der Proposition assertiert, wie bei modal einschränkenden oder negierenden Satzadverbien (vielleichtlangeblich/möglicherweise) und Prädikaten des Typs wie in (42) und (43) (Es ist möglich/es kann seinlfalsch ist ...) der Fall, sondern es handelt sich quasi um eine „Setzung“ einer alternativen Welt, bezogen auf die der Sprecher seine Behauptung als wahr beansprucht.

Und genau in dieser Komponente der „Setzung“ einer alternativen Welt unterscheiden sich auch die V2-Einbetter von den Standardkonditionalen. Hier wird nicht mit dem V2-Satz eine Bedingung gesetzt, unter der die Proposition des externen Arguments wahr ist, sondern eine fiktive Realität, eben eine alternative Welt, etabliert. Mit einem V2-Einbetter fordert der Sprecher gewissermaßen den Hörer auf: „Nimm mal an/stell dir vor, p ist der Fall“; in den V2-Einbettern angenommen, gesetzt (den Fall), unterstellt ist dies auch wörtlich ausgedrückt.

Wenngleich also die V2-Einbetter semantisch die Bedingungen für V2Komplementsätze erfüllen (schließlich sind sie ohnehin sämtlich deverbale Bildungen), verhalten sie sich syntaktisch anders als V2-Subjunktoren oder desintegrierte Adverbkonnektoren. Sie betten ihr internes Komplement ein, bilden also mit diesem zusammen eine Phrase, die genauso wie eine VL-Subjunktorphrase selbst im Vorfeld eines V2-Satzes eingebettet werden kann.

(44) Unterstellt, es kommt in Kiel zu einer rot-grünen und in Mainz wieder zu einer sozialliberalen Koalition, ändert sich an den Verhältnissen aber nichts, soweit es um die klassischen Konfrontationslinien geht. (Mannheimer Morgen, 25.03.1996)

Interessanterweise treten nun aber die anteponierten V2-Einbetterphrasen meist gerade nicht wie oben, sondern typischerweise entweder wie sententiale Sprechaktadverbiale und Irrelevanzkonditionale ihrerseits desintegriert oder aber in einer Linksversetzungskonstruktion mit einem resumptiven dann oder so auf.

(45) Gesetzt den Fall, die Deutsche Telekom hätte es ebenso gemacht - es hätte einen empörten Aufschrei durch alle deutschen Wirtschaftsredaktionen gegeben. (Mannheimer Morgen, 30.03.2000) 
(46) Angenommen, Sie spielen in $\mathrm{H}$ das Kreuz-Hand Spiel, so müssen Sie, wenn $\mathrm{V}$ und $\mathrm{M}$ klug und richtig spielen, mit 39 Augen ihr Spiel verlieren. (Mannheimer Morgen, 10.06.89)

V2-Einbetter sind somit am besten als „kategoriale Zwitter, die sowohl Eigenschaften eines Konnektors als auch Eigenschaften eines Prädikatsausdrucks aufweisen“" zu beschreiben (HDK 2003, S. 446). Von der Nähe zum Prädikatsausdruck zeugen auch die für einbettende Konnektoren untypische Interpunktion, die adverbiale Modifizierbarkeit (mal angenommen) und die Existenz komplexer VL-Subjunktoren: gesetzt den Fall, dass; vorausgesetzt, dass.

Als Matrixsatzprädikate von V2-Sätzen treten übrigens auch keine Ereignisprädikate auf, wenngleich diese dass-Sätze einbetten können. Auch dies findet eine Entsprechung im Ausschluss von desintegrierten temporalen Konnektoren und Adverbialsätzen.

(47a) *Es kommt vor/geschieht/passiert, einer schummelt und gewinnt.

(47b) *Daraufhin/dann: das Spiel wird annulliert.

(47c) *Als Hans schummelte: das Spiel wurde annulliert.

\subsection{Ein Vorschlag für die satzsyntaktische} und semantische Analyse der Nullstelle

Das Auftreten von Konnektoren und Adverbialsätzen an der Nullstelle unterliegt semantischen Bedingungen:

(i) Die Restriktionen sind in etwa die gleichen wie die bei der Einbettung von V2-Komplementsätzen unter ein Matrixsatzprädikat.

(ii) Der auf einen Konnektor in Nullstelle folgende Satz darf nicht von außen in seinen Wahrheitsbedingungen beeinflusst werden. (Das erklärt, weshalb temporale und konditionale Adverbien und Adverbialsätze in der Nullstelle nicht möglich sind.) Er darf aber auch keinen Einfluss auf die Wahrheitsbedingungen des Vorgängersatzes haben. (Das erklärt die Nicht-Existenz temporaler und konditionaler V2-Subjunktoren.) In einer Struktur aus Matrixsatz und Komplement wäre dies gleichbedeutend mit einem Hineinregieren "nach links" in die Matrixsatzstruktur bzw. über diese hinweg, - syntaktisch ein Unding.

(iii) Ein auf einen Konnektor an der Nullstelle folgender deklarativer V2Satz ist assertierend bzw. ,vermittelt" assertierend und darf nicht präsupponiert sein. (Das erklärt die Nicht-Existenz von $d a-\mathrm{V} 2$ und den Ausschluss von nicht-fokalen V2-Sätzen nach V2-weil bzw. denn.) Er ist eine ,eigene Mitteilungseinheit mit eigener Fokus-Hintergrund-Struktur, die gleichgewichtig neben der Informationsstruktur des ,Matrixsatzes“ steht." (Altmann 1997, S. 75). 
(iv) Adverbkonnektoren, Subjunktoren und Adverbialsätze an der Nullstelle haben tendenziell eine nicht-propositionsbezogene (einstellungsoder äußerungsbezogene) Lesart. Die Nullstelle hat Disambiguierungsfunktion, wenn ein Konnektor eine propositionsbezogene und eine nicht-propositionsbezogene Interpretation hat (s.a. FabriciusHansen 2007, S. 769; dagegen sprechen ihr Thim-Mabrey 1985 und Ortner 1983 grundsätzlich metakommunikativen Status zu).

Damit lassen sich Konnektoren und Satzadverbiale an der Nullstelle satzsyntaktisch auch in der Form von Matrixsatzstrukturen mit abhängigen V2-Sätzen modellieren, - ein Übergangsphänomen zwischen Parataxe und Hypotaxe. ${ }^{9}$ Sie sind durch Hinzufügung eines redundanten Prädikats (der reinen Assertionsbestätigung gilt (außerdem/weillwährend + gilt)) zu einer vollständigen Matrixsatzstruktur ausbaubar. Solche Strukturen sind auch tatsächlich belegt:

(48) Solidarität beim Sparen kann der Senat den Berlinern nur abfordern, wenn dieser teure Unfug ein Ende hat. Schließlich gilt: Sparen fängt von oben an. (die tageszeitung, 30.01.1996, S. 25)

(49) Der Goldpreis ist so niedrig wie seit 1979 nicht mehr. Dennoch gilt: Das glitzernde Geschäft geht weiter, 130 Jahre, nachdem der erste Diamant am Oranjefluß gefunden wurde. (Die Presse, 03.01.1998)

Bei nicht-propositionalem Bezug kann, nach dem Muster der oratio recta nach verba dicendi, der Illokutionstyp propositionalisiert werden: Deshalb/ ergo/weil du mich fragst + behauptelfragelbefehle ich (ähnlich bereits bei ThimMabrey (1985), und in der GDS (1997, S. 2306)). Dagegen ist die Ergänzung eines Geschehensprädikats, wie sie Temporalkonnektoren fordern würden, nicht möglich, da diese keine V2-Sätze einbetten können.

Diese Modellierung erhebt nicht den Anspruch einer Nachbildung einer sprachlichen Realität oder diachronen Entwicklung, wenngleich bekanntlich verkürzte, idiomatisierte Matrixsatzprädikate sich zu Konnektoren (es sei denn, geschweige denn, gesetzt den Fall, mal angenommen, nebenbei gesagt)

9 Reis (1997) unterscheidet an einer Stelle „normale“ abhängige V2-Sätze von sog. Doppelpunkt-V2-Sätzen, die sich von ersteren u.a. prosodisch und durch den nichtkonjunktivischen Modus unterscheiden, geht aber in der Folge auf diesen Typ nicht weiter ein und bezieht ihre Ausführungen im Wesentlichen nur auf V2-Sätze nach verba dicendi und putandi. Eine scharfe Abgrenzung dieser zwei Typen von abhängigen V2Sätzen scheint mir allerdings zweifelhaft. Plausibler dürfte es sein, mit ebensolchen Übergängen zwischen Abhängigkeit/Hypotaxe und Nicht-Abhängigkeit/Parataxe bei V2-Sätzen unter Matrixsatzprädikaten zu rechnen, wie im Konnektorensystem mit Übergängen zwischen Integration und Desintegration gerechnet werden muss (wie oben für den Fall der V2-Einbetter dargestellt). Die Grauzonen im System der Konnektoren würden dann genau die Grauzonen zwischen Hypotaxe und Parataxe bei der Komplementsatzeinbettung spiegeln. 
oder konnektorartigen Einheiten (hinzukommt, zugegeben, Einwand, Problem, Grund, Fazit, im Gegenteil) und Diskursmarkern (mein'ich, find'ich, glaub'ich) entwickeln können. Damit kommt der Nullstelle für Konnektoren auch eine Art Katalysatorfunktion zu. Die „Rückführung“ von Konnektoren an der Nullstelle auf Komplementsatz einbettende Matrixsätze sollte aber nicht als Ellipse im Sinne der Dudengrammatik (Duden 1995, S. 761) verstanden werden, wo weil-V2 als verkürztes weil Folgendes der Fall ist analysiert wird. Hier wird Ellipse erstens als eine Art Defizit der gesprochenen Sprache in einen normativen Kontext gerückt, und zweitens wird sie offenbar als irgendwie „reales" sprachliches Phänomen angesehen.

Eine solche Vorstellung steht wohl auch hinter der teilweise recht harsch vorgetragenen Kritik der Gesprächsforschung, der Ellipsenlösungen meist als Kapitulation einer nicht gegenstandsadäquaten, satzzentrierten Grammatikographie gelten. Dagegen ist einzuwenden: Erstens sind die hier angesetzten Ellipsen nicht willkürlich, und sind, auch wenn sie anders als Koordinationsreduktionen nicht mit Material aus dem Kontext gefüllt werden, sprachökonomisch plausibel: Auch in anderen Konstruktionstypen des Deutschen kann ein semantisch „armes“ Prädikat wie die Kopula weggelassen werden, und in Sprachen wie dem Russischen wird sie im Präsens überhaupt nicht kodiert.

(50) Bei offenem Bruch nur reponieren, wenn ohne große Schmerzen und Stress möglich. (http://www.jgvnordhessen.de/lhilfe.html)

(51) Ich hatte, weil blond, die sticksüchtige Streberin Mary zu mimen, älteste Tochter und öder Ableger von Moralapostel Charles und Mutterpflanze Caroline. (die tageszeitung, 18.08.1997, S. 14)

Zweitens lässt sich damit die Nullstelle satzsyntaktisch problemlos einordnen - Komplementsatzeinbettung unter einen Matrixsatz kann jede syntaktische Theorie darstellen. Und drittens lassen sich nur so die semantischen Restriktionen an der Nullstelle systematisch erklären und auf ein Prinzip zurückführen, das auch in anderen Teilbereichen der Grammatik wirksam ist. Erklärungsmuster, die sich auf die Besonderheiten des sukzessiven Planungsprozesses bei der gesprochenen Sprache stützen, bleiben demgegenüber zu beliebig und zu wenig restriktiv. ${ }^{10}$

10 Die hier vorgeschlagene Analyse beschränkt sich auf Konnektoren und Adverbialsätze an der Nullstelle und schließt Herausstellungsstrukturen wie Freies Thema und Linksversetzung aus. Letztere tritt, insbesondere bei V2-Subjunktoren, nicht selten in Kombination mit konnektoraler Nullstellenbesetzung auf, vgl. Bsp. (17) und (19). Überdies sind die prosodischen, syntaktischen und semantischen Eigenschaften von Linksversetzungen so verschieden von den hier diskutierten Fällen, dass eine Subsumtion der beiden Phänomene unter ein Konzept und der Versuch einer einheitlichen funktionalen Erklärung für beide methodisch wenig fruchtbar sein dürfte. 


\section{Vorteile der Nullstelle}

Das Auftreten von Konnektoren an der Nullstelle ist teilweise „normativ diskriminiert" (Sandig 1973), ist aber weit verbreitet bzw. in (räumlicher und medialer) Ausdehnung begriffen. Ausgehend von einer Art „sprachdarwinistischem Credo", wonach eine vom Standard abweichende Variante sich nur dann neben dem Standard halten oder gegenüber diesem durchsetzen kann, wenn sie für Sprecher und/oder Hörer zusätzliche Vorteile bringt, ist zu fragen, was die Nullstelle, die ja auch beim einfachen Satz existiert, gerade für die satzverknüpfenden Konnektoren so attraktiv macht.

(i) Ikonizität der Form zur semantischen Hierarchie

Der desintegrierte Ausdruck liefert eine „Interpretationsanweisung“ für die Nachfolgeäußerung (Auer 1997, Fiehler et al. 2004). Die Linearstruktur ist damit ikonisch zur semantischen Hierarchie. Für Konnektoren heißt dies: frühestmögliche Information des Hörers über die Art des Anschlusses zum Vortext. Damit kommt die Struktur auch dem sukzessiven Planungsprozess in der gesprochenen Sprache entgegen, wo sie bekanntlich sehr frequent ist. Dem Sprecher liefert sie Planungszeitgewinn, dem Hörer mehr Transparenz.

(ii) Disambiguierungspotential; Kennzeichnung von nicht-propositionalem Bezug

Aus der V2-Stellung kann - anders als bei VL - auf eigenständiges Illokutionspotential geschlossen werden. Nun haben viele Konnektoren und Adverbialsätze neben einer propositionsbezogenen auch eine nicht-propositionsbezogene Lesart; hier kann die Nullstelle zur Disambiguierung genutzt werden.

\section{(iii) Größerer informationsstruktureller Freiraum}

Das zusätzliche linke Außenfeld eröffnet Gestaltungsmöglichkeiten für Topikalisierungs- und Fokussierungsverfahren, die VL- und einfache V2-Sätze nicht haben; es werden sogenannte "main clause phenomena" wie Linksversetzung, I-Topikalisierung und kontrastive Topiks, VP-Voranstellung u.ä. möglich (s. Green 1976, Günthner 1993, S. 48f., Uhmann 1998, S. 111 f.). Tatsächlich treten V2-Subjunktoren oft zusammen mit Linksversetzung auf. Der Konnektor kann an der Nullstelle hervorgehoben und dadurch die Relationierung selbst stärker profiliert und „der Übergang als solcher herausgestellt" werden (GDS 1997, S. 2390), ohne dass der Konnektor selbst den Nuklearakzent tragen muss; der nachfolgende Satz kann fokal bleiben. Effekte wie die folgenden lassen sich mit einer VL-Struktur nicht oder nur schwer erzielen.

(52a) Desintegration ist praktisch, weil, so ein zusätzliches /FELD, das kann man IMmerl brauchen. (kontrastive Topiks, Linksversetzung) 
(52b) Desintegration ist wichtig, weil, verLOren\wären wir ohne sie // was TÄten\wir nur ohne sie! (Fokusvoranstellung, Emphase)

(52c) Desintegration ist praktisch, weil, wenn man ein zusätzliches Feld hat, kann man auch viel Komplexeres unterbringen.

Fazit: Die Besetzung der Nullstelle durch Konnektoren ist a) vollkommen systemkonform und b) vorteilhaft, und auf dieser Basis sollte sie auch bewertet werden.

\section{B Fehler im System: der Konjunktor sowohl ... als auch}

Die Vorstellung, dass eine Einzelsprache an einer bestimmten Stelle für ihre Sprecher zu „schwierig“ geworden sein könnte, spielt in der Flexionsmorphologie eine wichtige Rolle, etwa in Wolfgang Wurzels Sprachwandeltheorie einer allgemeinen „natürlichen“ Entwicklung hin zum Markiertheitsabbau. Am Beispiel des zweiteiligen additiven Konjunktors sowohl ... als auch soll gezeigt werden, dass man auch bei Konnektoren mit solch unbefriedigenden Ausgangszuständen rechnen muss. Was sind hier die Störfaktoren?

\section{Störfaktoren}

\subsection{Störfaktor 1: Unterschiedliche und unklare Wortart- zugehörigkeit der Bestandteile}

Bei korrelativen Konnektoren hat jeder Teil eine eigene syntaktische Charakteristik, nach der er in seinem Teilsatz verarbeitet werden muss. Diese ist aber bei den beiden Bestandteilen von sowohl ... als auch unterschiedlich und überdies unklar.

- Sowohl: kann im Mittelfeld stehen: ein Argument Pro AdverB und KonTrA KONJUNKTOR

(1a) Wir haben sowohl die Mädchen als auch die Jungen berücksichtigt.

- Es kann nicht im Vorfeld stehen: ein Argument konTra ADVERB und Pro KONJUNKTOR

(1b) ??Sowohl haben wir die Mädchen berücksichtigt als auch die Jungen.

- Es kann zusammen mit einer anderen Konstituente im Vorfeld stehen, und dabei zwischen Ante- und Postposition variieren, eine für FoKUSPARTIKelN typische Stellung.

(1c) Sowohl die Mädchen haben wir berücksichtigt als auch die Jungen.

(1d) Die Mädchen sowohl haben wir berücksichtigt als auch die Jungen.

- als (auch) erlaubt koordinativ gestützte Rückwärtsellipsen: ein Argument PRO KONJUNKTOR 
(2) Sowohl die Mädchen [...] als auch die Jungen haben wir berücksichtigt.

- Es kann Pluralkongruenz bei Subjektverknüpfung auslösen: ein Argument PRO KONJUNKTOR

(3) Sowohl Fritz als auch Hans besitzen ein Haus.

- Es lässt Vorwärtsellipsen zu, aber keine Ergänzung des elliptischen Koordinats zum Satz.

(2a) Sowohl die Mädchen haben wir berücksichtigt als auch die Jungen $[\ldots]$.

(2b) ? ... als auch haben wir die Jungen berücksichtigt. (Adverb-Muster)

(2c) ?... als auch wir haben die Jungen berücksichtigt. (Konjunktor-Muster)

(2d) ?... als auch wir die Jungen berücksichtigt haben. (Subjunktor-Muster)

1.2 Störfaktor 2: Defizite bei „langen Koordinationen“ (VP, Satz, Äußerung)

„Kurze Koordinationen“ von Material unterhalb der Satzebene (NP, PP, AdjP etc.) sind problemlos. Versucht man aber wie in (2), gemeinsames Material zu Sätzen zu expandieren, gerät man in die Bredouille. Finite Verben können nämlich nur in Verbletztstellung verknüpft werden (3a/4); Verbzweitsätze nur dann, wenn sie sich das Finitum teilen, sprich, wenn sie einen Verbalkomplex haben. (3b/5).

(3a) ... dass Hans den Apfel sowohl wäscht als auch schält.

(3b) Hans hat den Apfel sowohl gewaschen als auch geschält.

(4) Hier könnte eine behutsame Umstellung einzelner Komponenten auf ein steuerfinanziertes Modell dazu führen, dass sowohl die Unternehmen entlastet werden als auch die Arbeitnehmer mehr Geld in die Tasche bekommen. (die tageszeitung, 26.3.2005, S. 15)

(5) Im Kapital hat Marx sowohl den obigen Satz richtiggestellt wie die Umstände entwickelt, welche der kapitalistischen Produktion erlaulauben, den Preis der Arbeitskraft mehr und mehr unter ihren Wert zu drücken. (mew/WAC.03005, S. 83)

Koordinationen von vollständigen Verbzweitsätzen hingegen sind in allen topologischen Varianten problematisch. Es gibt also kein kanonisches, normativ unbedenkliches Muster für die Koordination von Verbzweitsätzen mit sowohl ... als auch. 
(3c) ?Hans wäscht sowohl den Apfel als auch schält (er) ihn.

(3d) ?Sowohl Hans wäscht den Apfel als auch (er) schält ihn.

(3e) ?Sowohl wäscht Hans den Apfel als auch schält (er) ihn.

(3f) ?Sowohl bäckt Hans einen Apfelkuchen als auch macht Maria Kaffee.

\subsection{Störfaktor 3: Widerspruch zwischen semantischer und syntaktischer Strukturanforderung}

Die semantische Relation Additivität wird übereinzelsprachlich typischerweise koordinativ und nicht subordinativ kodiert (vgl. Haspelmath 2004). Koordination verlangt Parallelstruktur der Koordinate wie in (6a). Sowohl ... als auch aber geht auf eine Vergleichskonstruktion zurück - und die wird im heutigen Deutsch subordinativ und damit asymmetrisch kodiert wie in (7b).

(6a) Hans schält die Äpfel und Maria rührt den Teig.

(6b) *Hans schält die Äpfel und Maria den Teig rührt.

(7a) *Hans schält die Äpfel viel sorgfältiger als Maria rührt den Teig.

(7b) Hans schält die Äpfel viel sorgfältiger als Maria den Teig rührt.

\subsection{Störfaktor 4: Asynchronien und historische Relikte}

Die historische Entwicklung kann man - sehr vergröbert - beschreiben als einen unvollständigen Übergang von einer Vergleichskonstruktion zu einer Koordinationskonstruktion (s. Breindl 2007 und 2008, Eggs 2006). Das als bewahrt hier außerdem noch die alte (heute noch in den Dialekten lebendige) Positivmarkierung, die es vor der Verschiebung im System der Vergleichskonstruktionen innehatte, die einsetzte, nachdem der ursprüngliche Komparativmarker danneldenne sich zum Kausalkonnektor entwickelte.

Positiv
ahd./mhd. wie $\rightarrow$ als
whd. $\rightarrow \begin{aligned} & \text { Komparativ } \\ & \text { als }\end{aligned} \rightarrow$ Kausalkonnektor

Dieser diachrone Übergangsstatus dürfte letztlich auch der Grund für die oben genannten drei synchronen Störfaktoren sein. Alles in allem ist sowohl ... als auch ein reichlich fehlerbehaftetes Objekt. Es gleicht einem Fahrzeug mit separaten Lenkungen für Vorderachse und Hinterachse, die beim Lenken in verschiedene Richtungen ziehen. Das geht gut, solange das Gelände keine Kurven aufweist. Auf kurvenreicher, sprich satzförmiger Strecke aber sind Unfälle vorprogrammiert. 


\section{Schadensbilanz: Reparaturversuche und grammatische Totalschäden}

Wie gehen nun Sprecher mit diesem fehlerhaften Produkt um? Zunächst: sie meiden kurvenreiche Strecken. Eine Stichprobe in den Mannheimer Korpora geschriebener Sprache ergab $90 \%$ Verwendungen in kurzen Koordinationen; in der gesprochenen Sprache ist der Konnektor ohnehin selten. Generell versuchen Sprecher, sich möglichst an Muster anzulehnen. In den Belegen ließen sich fünf jeweils gut belegte Muster erkennen, die hier nach aufsteigendem Schwierigkeitsgrad geordnet sind.

\subsection{Umkategorisierung zum einteiligen Konjunktor: Muster und}

Nach Aufweis der Beleglage scheint nicht wenigen Sprechern der zweiteilige Konnektor generell zu kompliziert: Diese Vereinfacher unter den Sprechern bauen quasi die Vorderachslenkung aus und interpretieren sowohl als optionales Adverb - aufgrund der Mittelfeldfähigkeit zurecht. Das Ergebnis ist ein einteiliger Konjunktor als auch, der wie und funktioniert.

(8) Richter als auch Staatsanwalt lehnen zunächst eine Haftentlassung mit der Begründung einer möglichen Revision ab. (die tageszeitung, 17.12. 1986, S. 5)

(9) „Als ich in die Wohnung kam und den Reinhard auf dem Bett der Karola im Kinderzimmer antraf und die Melanie als auch die Karola am Arm ergriff und sie schüttelte, stellte ich fest, daß sie [...] tot waren." (Gerhard Mauz (2005): Die großen Prozesse der Bundesrepublik Deutschland. Hannover: zu Klampen, S. 190)

\subsection{Umkategorisierung zum einteiligen Adverbkonnektor: Muster ebenso, ferner}

Andere Sprecher, ebenfalls vom Typ der Vereinfacher, entledigen sich sozusagen der Hinterachslenkung, aber nicht genug damit: sie bauen nun die Vorderachslenkung nach hinten, - denn die scheint aufgrund ihrer adverbialen Charakteristik etwas flexibler als das als auch. Dieser Sprecher kategorisieren um zum einteiligen vorfeldfähigen Adverbkonnektor sowohl, nach dem Muster additiver Adverbkonnektoren wie ferner, ebenso, außerdem. Damit ist das Produkt kurventauglich bzw. satzverknüpfungstauglich.

(10) Die Gesellschaft haftet nicht für die Richtigkeit oder Verwendbarkeit der Preisspiele, sowohl haftet sie auch nicht für den Verlust oder entstandenen Schaden [...]. Sowohl kann die Gesellschaft die einzelnen Eigenschaften der Dienstleistung oder die ganze Dienstleistung begrenzen $[\ldots]$. (http://www.fangdirdenpreis.com/info_center.php?column= 1 \&content $=34$ ) 
(11) In dieser Option kann man SMS und MMS verschicken, sowohl kann man auch chatten und Sprachmitteilungen einstellen. (http://www.yopi.de/rev/265317)

(12) Man kann gegen das Programm Schach spielen und trainieren, sowohl kann man noch Schachpartien analysieren. (http://www.ciao.de/Fritz_8_Test_3062568)

\subsection{Vereinheitlichung der syntaktischen Charakteristik: symmetrisches Muster weder ... noch}

Ein von vielen Sprechern genutztes Muster ist die Angleichung der syntaktischen Charakteristik der beiden Bestandteile zu der von vorfeldfähigen Adverbien. (Das sind sozusagen die Bastler unter den Sprechern.) Dieses Muster ist von den fünf hier aufgezählten eines der häufigsten. Der Grund dürfte neben der allgemeinen Symmetrieanforderung additiver Satzverknüpfung auch der sein, dass das negative Pendant von sowohl ... als auch, das korrelative weder ... noch, nach genau diesem Muster funktioniert.

(13) Denn sowohl wird die Erschaffung der Welt in sechs Tagen vollendet, als auch vollzieht sich die Bewegung der Sonne von Wendekreis zu Wendekreis in sechs Monaten. (http://www.unifr.ch/bkv/kapitel272-5.htm)

(14) Sowohl ist die Reihenfolge des Auftretens der Kindelemente definiendum und definiens beliebig, als auch kann das definiens diskontinuierlich auftreten.

(http://www.hytex.uni-dortmund.de/)

(15) Sowohl ist die Wikipedia ein Erfolg, den ich nie vorher erahnt hätte als auch beruhen mehrere Jahre menschliche Entwicklung auf offenen Inhalten.

(de.wikipedia.org/wiki/Benutzer:Mathias_Schindler/OpenContentFragen/Southpark)

(16) Denn sowohl ist die Gleichung von Religion und begrifflicher Lehraussage aufgelöst, als auch ist die historische Ansicht der Bibel als eine Sammlung verschiedenster historischer Urkunden allgemein geworden. (Folkart Wittekind (2000): Geschichtliche Offenbarung und die Wahrheit des Glaubens. Mohr Siebeck: Tübingen, S. 93)

\subsection{Sowohl als Adverb; als auch als Konjunktor: asymmetrisches Muster entweder ... oder}

Komplizierter, aber immer noch an ein Muster angelehnt, nämlich das von entweder ... oder, ist die asymmetrische Lösung, bei der sowohl als Adverb 
interpretiert wird, als auch als voll funktionsfähiger Konjunktor. Alternativ kann bei dieser Lösung auch und an der Konjunktorstelle erscheinen.

(17) Da ist eben beides möglich im selben Zeitraum: sowohl ist „etwas“ als auch es wird „etwas“.

(http://www.foren4all.de/archive/index.php?t-5108-p-3.html)

(18) Sowohl wird die Liebe der Sprecherin zur Oper deutlich, als auch die Liebe der Zuhörenden zu Oper wird geweckt. (http://www.amazon.de/Oper-Eine-Liebeserk1\%C3\%A4rung-2$\mathrm{CDs} / \mathrm{dp} / 389830583 \mathrm{X})$

(19) Sowohl kann man sie dann weiterverschenken und persönlich brauche ich auch grad welche. (http://www.ureader.de/msg/16048732.aspx)

\subsection{Umkategorisierung von als zum Subjunktor: asymmetrisches Muster wie}

Beim Subjunktormuster gibt es Varianten mit Distanzstellung von als und auch - hier wird als an das subordinierende wie-Muster angelehnt -, aber auch die Interpretation als komplexer Subjunktor als auch.

(20) Diese Vorlesung bildet sowohl den Abschluss meiner Vorlesungsreihe zum „Islam in der Romania“ als sie auch eine eigenständige Einführung in die frankophonen Literaturen Afrikas darstellt. (http://www2.tu-berlin.de/fak1/)

(21) Der [...] Band „Kanonbildung bei audiovisuellen Medien im Deutschunterricht?" untersucht nun sowohl Sinn und Zweck des Filmkanons als er auch Unterrichtsbeispiele sowie didaktische Konzepte vorstellt [...]. (http://www.f-lm.de/?p=106)

(22) Die AGGPG distanziert sich theoretisch sowohl von Körper-, Rasseund Geschlechternormen als sie auch auf Mängel medizinischer und sozialkonstruktionistischer Diskurse hinweist. (http://www.stachel.de/00.01/1AGGPG.html)

(23) Denn sowohl wird jeder Fehler [...] gnadenlos offenbar, als auch jede stilistische „Falschheit" wahrgenommen wird. (http://www.joergbenner.de/Hobbies/Musik/musik.html)

\section{6 „Grammatische Totalschäden“}

Die Konstruktionstypen 2.1 bis 2.5, die in Analogie zu einem Muster gebildet sind, sind auch einigermaßen gut belegt. Dagegen haben die folgenden Bei- 
spiele großteils den Charakter von Einzelstücken. Ein gebräuchliches Strukturmuster, das zur Charakteristik der Bestandteile passen könnte, ist für sie nicht erkennbar.

In den folgenden drei Belegen nimmt sowohl die Nacherstposition zwischen einer Konstituente im Vorfeld und dem Finitum ein. Diese Stellungsvariante würde zwar zur Fokuspartikelhaftigkeit von sowohl passen (s. Bsp. 1c/d), allerdings ist die Vorfeldkonstituente hier gerade kein Koordinat, sondern gehört zum gemeinsamen Koordinationsrahmen.

(24) Der Cole2k Media Codec Pack in der hier angebotenen Advancedversion sowohl ist für die Wiedergabe als auch für die Neukodierung von Filmen und Musik gedacht.

(http://cole2k-media---codec-pack.softonic.de/ie/47440)

(25) Dies sowohl kann an den langatmigen Songs, wie auch an den langen Intros liegen, welche fast bei jedem Track anzureffen sind. (http://www.schwermetall.ch/cdkritiken/kritik454.php)

(26) Diese sowohl kann auf tatsächlich größer gewordene Menümengen zurïckgehen als auch auf größere Verluste während der Verarbeitung in der Lebensmittelindustrie und in den Haushalten sowie auf größeren Verderb von Lebensmitteln.

(http://www.ernaehrungswende.de/pdf/DP7_Szenarien_2005_final.pdf)

Die folgenden Beispiele zeigen nicht nachvollziehbare Aufspaltungen der Koordinate.

(27) Sowohl ist Berlin die bevölkerungsreichste Stadt [...], als mit einer Fläche von 89.169 ha auch die flächengrößte Stadt Deutschlands. (http://www.willischnitzler.de/reiselaender/staedte/berlin.htm)

(28) Sowohl kann Wiedergewinnung von unentdecktem Datumstext und Bildern leicht sein als auch tatsächlich von Software funktionieren. (http://treibsmitmir.de/Software/Shareware/Info/Drive_Recovery_ Software.html)

Die „Störungsanfälligkeit“ des Konnektors kann sich offensichtlich auch auf seine Kombinatorik auswirken:

(29) Fritz 8 ist ein weltbekanntes und weltgenutztes Schachprogramm, mit dem man sowohl alleine oder online gegen andere Spieler Schach spielen kann. (htp://www.ciao.de/Fritz_8_Test_3062568)

(30) Dieses Seminar betritt in mehrfacher Hinsicht Neuland, weil im Lehrangebot sowohl der westafrikanische Film, aber noch weitaus weniger der Film aus Afrika eine besondere Rolle spielen. (http://www2.tu-berlin.de/fak1/el/board.cgi?id=franzphil\&action= simple_view\&gul=168) 
(31) Ich bin auch bei AOL und konnte sowohl vor der RO-Downtime sowohl kann ich auch jetzt noch recht lagfrei spielen. (http://forum.rocards.de/index.php?page=Thread\&postID=651061)

Und im folgenden Beleg wird der Konnektor mehrfach auf unterschiedliche Weise normabweichend gebraucht; - ein „grammatischer Totalschaden“ an der Grenze zum kommunikativen Schaden.

(32) Untersuchungen die beinhalten HD, ED + Wirbelsäule röntgen, DNA Abnahme und Einlagerung, sowohl MDR1 Test. [...]. Die Zuchttauglichkeit auf Probe ist nur zu erlangen in Gegenwart von Zuchtgutachter, 2 Zuchtwarten, sowohl ist zulässig in Gegenwart des Zuchtgutachters, Zuchtwart und/oder Richter [...]. Nur nationale und Internationale Ahnentafeln von anerkannten Dachorganisationen/ Vereinen werden durch den BWS umgeschrieben, sowohl muss der Hund bei Gelegenheit dem Zuchtgutachter vorgestellt werden. (http://www.bund-weisser-schaeferhunde.info/index.php?page= Zuchtordnung)

\section{Weitere Indikatoren für Störungen}

Über diese normativ mehr oder weniger diskriminierten Verwendungen hinaus gibt es im Zusammenhang mit dem Konnektor sowohl ... als auch noch einige Phänomene, bei denen teilweise nicht so klar ist, inwieweit sie selbst als zusätzliche Auslöser oder vielmehr als Symptome für Störungen fungieren.

\subsection{Hoher formaler Variationsspielraum}

Der Konnektor hat einen ungewöhnlich hohen Variationsspielraum; er tritt in vier normativ nicht zu beanstandenden Formvarianten auf, hier nach ihrer Frequenz in den Mannheimer Korpora geordnet: sowohl p als auch q; sowohl $p$ wie $q$; sowohl $p$ als $q$; sowohl $p$ wie auch $q$.

Diese Variationsbreite unterstützt auf jeden Fall den in 1.4 erhobenen diachronen Befund, dass es sich um eine nicht zur Gänze grammatikalisierte Konstruktion handelt: Die Formvarianten repräsentieren dabei unterschiedliche Nähe zur Vergleichskonstruktion.

\subsection{Interpunktion}

Vom Konflikt zwischen subordinierender und koordinierender Struktur zeugen auch die Unsicherheiten bei der Interpunktion: in vielen Belegen findet sich vor als nach dem Muster subordinierter Nebensätze ein Komma (vg1. etwa die unterschiedliche Kommasetzung in ansonsten parallel gebauten Belegen: (15) vs. (16); (17) vs. (18); (20-22) vs. (23). Interessanterweise spiegelt sich die Hybridität auch in den einschlägigen Paragrafen des Regelwerks bzw. 
in deren Umsetzung im Duden, Bd. 9. Nach dem amtlichen Regelverzeichnis gilt:

Sind die gleichrangigen Teilsätze, Wortgruppen oder Wörter durch und, oder, beziehungsweiselbzw., sowie $(=$ und $)$, wie $(=$ und), entweder... oder, nicht ... noch, sowohl ... als (auch), sowohl ... wie (auch) oder durch weder ... noch verbunden, so setzt man kein Komma.

Dagegen vermerkt Duden Bd. 9: „Ist sowohl ... als auch mit Nebensätzen verbunden, werden immer Kommas gesetzt.“

(33) Er behauptet sowohl, dass er ein guter Stürmer ist, als auch, dass er das Tor hüten könne. (Duden, Bd. 9, ${ }^{5} 2001$, S. 782; identisch ${ }^{72007, ~ S . ~ 833) ~}$

\subsection{Schwankungen bei der Subjekt-Verb-Kongruenz}

Mit sowohl ... als auch verknüpfte Subjekte treten mit Schwankungen beim Numerus am Finitum auf: nach Duden Bd. 9 (2001, S. 782) ist der Singular hier ,zulässig“. Das liegt an der obligatorisch distributiven Verrechnung der Prädikate mit den Koordinaten (Bsp. 34) und dem daraus resultierenden Ausschluss kollektiver Prädikate (35), ein Differenzmerkmal zwischen sowohl ... als auch und und (s. Breindl 2007 und 2008). Diese distributive Verrechnung geht ihrerseits wieder auf das Konto der Vergleichsstruktur-Relikte bei diesem Konnektor.

\#Sowohl Karl Marx als auch Friedrich Engels schrieben das Kommunistische Manifest.

*Verrühren Sie sowohl das Mehl als auch den Zucker miteinander.

\subsection{Grammatikographische Defizite}

Die Beschreibung von sowohl ... als auch in Grammatiken und Wörterbüchern ist häufig entweder zu restriktiv oder zu liberal. So würde die Generalisierung bei Redder (2007, S. 483), Konjunktoren seien „im Falle zweiteiliger Konjunktoren jeweils im Vorfeld" positioniert, das in Abschnitt 2.3 beschriebene symmetrische Adverbmuster als einziges Muster kanonisieren und damit Konstruktionen wie die folgenden erlauben

(36a) *Weder Hans hat Äpfel geschält noch Maria hat Kuchen gebacken.

(36b) *Sowohl Hans hat Äpfel geschält als auch Maria hat Kuchen gebacken.

Auch die in Buscha (1989, S. 115) angegebene Restriktion, sowohl ... als auch könne nur dann Sätze verbinden, ,wenn diese mindestens ein SG [= Satzglied] gemeinsam haben, das im zweiten Teilsatz getilgt ist", ist einerseits zu restriktiv, da Nebensatzkoordination ausschließend (weil sowohl Hans als auch Fritz das Buch gelesen hat) andererseits mit dem Hinweis auf ein beliebiges zu 
teilendes Satzglied zu vage, da die entscheidende Restriktion die ist, dass im zweiten Teilsatz kein finites Verb in Zweitstellung auftreten darf. Umgekehrt schließt die Behauptung in Hentsche1/Weydt (2003, S. 291), sowohl ... als auch könne Sätze nur dann verknüpfen, ,wenn es sich um gleichgeordnete Nebensätze handelt", möglicherweise wiederum Koordinationen wie Hans hat sowohl die Äpfel geschält als auch einen Kuchen gebacken aus. Detaillierte Beschreibungen, die die Restriktionen bei der Satzkoordination korrekt erfassen, finden sich vor allem in der GDS (1997, S. 2399) und im HDK (2003, S. $473 \mathrm{ff}$.).

Fazit: Als Produkt ist sowohl ... als auch so störanfällig, dass ein verantwortungsbewusster Hersteller, gäbe es ihn denn, im Grunde diesen Konnektor via Rückrufaktion aus dem Verkehr ziehen oder zumindest einen Warnhinweis anbringen müsste: „Nicht für Satzkoordination geeignet“. Zum Glück gibt es diesen Hersteller aber nicht, und Linguisten und Laien können sich weiterhin an den kreativen Lösungen der Sprecher erfreuen und darüber streiten, welcher Reparaturstrategie mehr Chancen einzuräumen sind. Wie auch immer man die hier angeführten Belege mit sowohl ... als auch bewerten mag, so sollte man nach dem hier Dargelegten den Schreibern zumindest mildernde Umstände zubilligen.

\section{Quellen}

Die mit Siglen gekennzeichneten Belege wurden den Mannheimer Korpora geschriebener Sprache (https://cosmas2.ids-mannheim.de/cosmas2-web/) und der Datenbank Gesprochenes Deutsch (http://dsav-oeff.ids-mannheim.de/) entnommen.

\section{Literatur}

Altmann, Hans (1997): Verbstellungsprobleme bei subordinierten Sätzen in der deutschen Sprache. In: Dürscheid, Christa/Ramers, Karl Heinz/Schwarz, Monika (Hg.): Sprache im Fokus. Festschrift für Heinz Vater zum 65. Geburtstag. Tübingen: Narr. S. 69-84.

Auer, Peter (1998a): Formen und Funktionen der Vor-Vorfeldbesetzung im gesprochenen Deutsch. In: Schlobinski, Peter (Hg.): Syntax des gesprochenen Deutsch. Opladen: Westdeutscher Verlag. S. 55-91.

Auer, Peter (1998b): Zwischen Parataxe und Hypotaxe. 'Abhängige Hauptsätze' im Gesprochenen und Geschriebenen Deutsch. In: Zeitschrift für Germanistik Linguistik. S. 284-307.

Breindl, Eva (2007): Additive Konjunktoren und Adverbien im Deutschen. In: Buscha, Joachim/Freudenberg-Findeisen, Renate (Hg.): Feldergrammatik in der Diskussion. Funktionaler Grammatikansatz in Sprachbeschreibung und Sprachvermittlung. Frankfurt a. M. u.a.: Lang. S. 141-164.

Breindl, Eva (2008): Sowohl Semantik als auch Pragmatik. Zur Interaktion sprachlicher Ebenen bei additiven Konjunktoren des Deutschen (und, sowohl als auch, sowie). In: Pohl, Inge (Hg.): Semantik und Pragmatik - Schnittstellen. Frankfurt a.M. u.a.: Lang, S. 253-281. 
Breindl, Eva/Waßner, Ulrich (2006): Syndese und Asyndese. Konnektoren und andere Wegweiser für die Interpretation semantischer Relationen in Texten. In: Blühdorn, Hardarik/Breind1, Eva/Waßner, Ulrich Hermann (Hg.): Text - Verstehen. Grammatik und darüber hinaus. Berlin/New York: de Gruyter. S. 46-70.

Buscha, Joachim (1989): Lexikon deutscher Konjunktionen. Leipzig: Enzyklopädie.

D'Avis, Franz Josef (2004): In front of the prefield - inside or outside the clause? In: Lohnstein, Horst/Trissler, Susanne (Hg): Syntax and Semantics of the Left Periphery. Berlin: de Gruyter. S. 139-177.

Duden (1995): Grammatik der deutschen Gegenwartssprache. 5. Auflage. Mannheim u.a.: Dudenverlag.

Duden (2005): Die Grammatik. Unentbehrlich für richtiges Deutsch. 7. Auflage. Mannheim u. a.: Dudenverlag.

Duden (2001): Richtiges und gutes Deutsch. Wörterbuch der sprachlichen Zweifelsfälle. 5. Auflage. Mannheim u.a.: Dudenverlag.

Duden (2007): Richtiges und gutes Deutsch. Wörterbuch der sprachlichen Zweifelsfälle. 7. Auflage. Mannheim u.a.: Dudenverlag.

Eggs, Frederike (2006): Die Grammatik von als und wie. Tübingen: Narr.

Eisenberg, Peter (1999): Grundriß der deutschen Grammatik. Band 2: Der Satz. Stuttgart/Weimar: Metzler.

Fabricius-Hansen, Cathrine (2007): Subjunktor. In: Hoffmann, Ludger (Hg.): Handbuch der deutschen Wortarten. Berlin/New York: de Gruyter. S. 759-790.

Fiehler, Reinhard et al. (2004): Eigenschaften gesprochener Sprache. Theoretische und empirische Untersuchungen zur Spezifik mündlicher Kommunikation. Tübingen: Narr.

GDS = Zifonun, Gisela/Hoffmann, Ludger/Strecker, Bruno et al. (1997): Grammatik der deutschen Sprache. Berlin/New York: de Gruyter.

Green, Giorgia (1976): Main Clause Phenomena in subordinate clauses. In: Language 52, S. $382-397$.

Haspelmath, Martin (2004): Coordinating constructions: an overview. In Haspelmath, Martin (ed.): Coordinating constructions. Amsterdam: John Benjamins. S. 3-39.

HDK = Pasch, Renate et al. (2003): Handbuch der deutschen Konnektoren. Linguistische Grundlagen der Beschreibung und syntaktische Merkmale der deutschen Satzverknüpfer. Berlin/New York: de Gruyter.

Johannessen, Janne Bondi (1998): Coordination. New York: Oxford University Press.

Karhiaho, Izabela (2003): Der Doppelpunkt im Deutschen. Göteborg: Acta Univ. Gothoburgensis.

Klein, Wolf Peter (in diesem Band): Sprachliche Zweifelsfälle

König, Ekkehard/van der Auwera, Johan (1988): Clause Integration in German and Dutch conditionnals, concessive conditionals, and concessives. In: Haiman, John/Thompson, Sandra A. (Hg.): Clause combining in grammar and discourse. Amsterdam/ Philadelphia: Benjamins. S. 101-133.

Meinunger, André (2004): On certain adverbials in the German 'Vorfeld' and 'Vor-vorfeld'. In: Sprache \& Pragmatik 52, S. 64-78.

Merkle, Ludwig (1993): Bairische Grammatik. München: Hugendubel.

Oppenrieder, Wilhelm (1991): Von Subjekten, Sätzen und Subjektsätzen. Untersuchungen zur Syntax des Deutschen. Tübingen: Niemeyer.

Pasch, Renate (1983): Die Kausalkonjunktionen da, denn und weil: drei Konjunktionen drei lexikalische Klassen. In: Deutsch als Fremdsprache 20/6, S. 332-337.

Pasch, Renate (1997): Weil mit Hauptsatz - Kuckucksei im denn-Nest. In: DS 25, S. 75-85. Pittner, Karin (1999): Adverbiale im Deutschen. Tübingen: Stauffenburg. 
Redder, Angelika (2007): Konjunktor. In: Hoffmann, Ludger (Hg.): Handbuch der deutschen Wortarten. Berlin/New York: de Gruyter. S. 483-524.

Reis, Marga (1993): Satzfügung und kommunikative Gewichtung. Zur Grammatik und Pragmatik von Neben- vs. Unterordnung am Beispiel ,implikativer“ 'und'-Konstruktionen im Deutschen. In: Reis, Marga (Hg.): Wortstellung und Informationsstruktur. Tübingen: Niemeyer. S. 203-249.

Reis, Marga (1997): Zum syntaktischen Status unselbständiger Verbzweit-Sätze. In: Dürscheid, Christa/Ramers, Karl-Heinz/Schwarz, Monika (Hg.): Sprache im Fokus. Tübingen: Niemeyer. S. 130-144.

Sandig, Barbara (1973): Zur historischen Kontinuität normativ diskriminierter syntaktischer Muster in spontaner Sprechsprache. In: DS 3, S. 37-57.

Scheutz, Hannes (1998): weil-Sätze im gesprochenen Deutsch. In: Hutterer, Claus/Pauritsch, Gertrude (Hg.): Beiträge zur Dialektologie des ostoberdeutschen Raumes. Göppingen: Kümmerle. S. 85-112.

Schröder, Peter (2006): Das Vorvorfeldkonzept aus gesprächsanalytischer Sicht. Plädoyer für eine handlungsorientierte Einheitenbildung in einer Grammatik der gesprochenen Sprache. In: Deppermann, Arnulf/Fiehler, Reinhard/Spranz-Fogasy, Thomas (Hg.): Grammatik und Interaktion. Radolfzell: Verlag für Gesprächsforschung. S. 203-243.

Selting, Margret (1999): Kontinuität und Wandel der Verbstellung von ahd. wanta bis gwd. weil. Zur historischen und vergleichenden Syntax der weil-Konstruktionen. In: Zeitschrift für Germanistische Linguistik 27/2, S. 167-204.

Thim-Mabrey, Christiane (1985): Satzkonnektoren wie allerdings, dennoch und übrigens. Stellungsvarianten im deutschen Aussagesatz. Frankfurt a. M. u.a.: Lang.

Uhmann, Susanne (1998): Verbstellungsvariation in weil-Sätzen: Lexikalische Differenzierung mit grammatischen Folgen. In: Zeitschrift für Sprachwissenschaft 17/1, S. 92 139.

Volodina, Anna (2007): Konditionale und kausale Relationen im gesprochenen Deutsch. Diss. Heidelberg.

Waßner, Ulrich H. (i.Dr.): Grammatical variation within Near-Standard German: connectors in corpora. Erscheint in: Proceedings of the $2^{\text {nd }}$ International Conference Grammar and Corpora, 25-27 September 2007, Liblice near Prague. Praha: Ústav pro jazyk ceský.

Wegener, Heide (1993): „Weil - das hat schon seinen Grund“. Zur Verbstellung in Kausalsätzen mit ,weil“" im gegenwärtigen Deutsch. In: DS 21, S. 289-305.

Wöllstein, Angelika (2008): Konzepte der Satzkonnexion. Tübingen: Stauffenburg. 